\section{CORONARY HEART DISEASE MORTALITY IN THE} NETHERLANDS FROM 1972 TO 2007

doi:10.1136/jech.2010.120956.6

${ }^{1}$ V Vaartjes, ${ }^{2} \mathrm{M}$ O'Flaherty, ${ }^{1} \mathrm{M}$ Bots, ${ }^{2} \mathrm{~S}$ Capewell. ${ }^{1}$ Julius Center for Health Sciences and Primary Care, University Medical Center Utrecht, Utrecht, The Netherlands; ${ }^{2}$ Department of Public Health, University of Liverpool, Liverpool, UK

Background Coronary heart disease (CHD) mortality has steadily declined since the early 1970s in The Netherlands. Recent work suggests that in some Western countries the rate of decline in some groups has begun to decrease and may be starting to plateau or even reverse. These changes may be a result of changes in the pattern for major cardiovascular risk factor trends.

Methods Data for all deaths between 1972 and 2007 in The Netherlands were grouped by year, sex, age at death and contemporaneous ICD code for CHD as cause of death. Per age-sex-group a joinpoint regression was fitted to detect points in time at which significant changes in the trends occur. For every period the linear slope of the trend, p-value, observed number of deaths (min-max) and observed CHD mortality rates (min-max) were tabulated. Furthermore we calculated the change in observed CHD mortality rate per period.

Results Between 1972 and 2007, the age-adjusted CHD mortality rates decreased overall by $75.6 \%$ in men and by $75.7 \%$ in women. In men aged 35-54 the decline in CHD mortality rate attenuated in the period 1993-1999 (change in CHD mortality rate in period 1980-1993: -0.53 and in period 1993-1999: -0.16). In women aged 35 to 54 the decline in CHD mortality rate attenuated in the period 1989-2000 (change in CHD mortality rate in period 1979-1989: -0.58 and in period 1989-2000: -0.02). After 1999-2000 CHD mortality rate further declined in both gender (in men change in $\mathrm{CHD}$ mortality rate in period 1999-2007 was -0.46, in women change in CHD mortality rate in period 2000-2007 was -0.38).

Conclusions Evidence from several Western countries suggests some levelling out of CHD mortality rates among younger men and women and warn that CHD mortality rates in these groups may be starting to increase. In the present study attenuation of the decline in CHD mortality among men and women aged $35-54$ years has been observed starting in the mid 90s. Furthermore, we observed that after a period where the decline in CHD mortality was attenuated, an increase in the pace of decline was observed. In order to explain these recent changes in $\mathrm{CHD}$ mortality rates, a detailed analysis of recent changes in cardiovascular risk factors is urgently required.

\section{ASSOCIATIONS BETWEEN MARITAL STATUS AND SURVIVAL AFTER A FIRST ACUTE MYOCARDIAL INFARCTION IN SCOTLAND, 1988-2004}

doi:10.1136/jech.2010.120956.7

C A Davies, A H Leyland. MRC Social and Public Health Sciences Unit, Glasgow, UK

Background There is evidence that marriage has a beneficial effect on health; unmarried experience higher mortality and incidence of mental and physical disorders. In particular, being unmarried is associated with elevated coronary heart disease mortality.

Objectives Investigate the association between marital status and survival after a first acute myocardial infarction (AMI) and any trends and relationships with age, sex and deprivation.

Design Linked hospital discharges and death records for all AMI events from 1981 to 2004 for those aged 30+ years. Case-fatality was divided into CF0: death on day of first AMI; CF1: death in 2-28 days following first AMI; and CF2: death in 29-365 days. Area deprivation (DEPCAT) was assessed using Carstairs scores. Marital status was categorised into married, never-married/ widowed and other.
Setting Scotland, population 5.1 million.

Main outcome measures Directly age standardised case-fatality rates were calculated. Odds of case-fatality by marital status, adjusting for age, sex, year, and area deprivation were estimated through multilevel logistic regression.

Results Between 1988 and 2004, 178781 (48\%) of the 372349 patients with a first AMI died on the day of event, 34198 (18\%) of those surviving the day of their first AMI died within 28 days and $17971(11 \%)$ of those surviving 28 days after their first AMI died within 1 year. Marital status was significantly associated with each case-fatality outcome. The odds of CF0 for never-married/widowed compared to married increased over time and was strongest in 60-74 year-olds living in deprived areas-for example, OR for men aged 60-74 in most deprived areas in 2000-2004 was 2.81 (95\% CI 2.65 to to 2.98 ) and for men $30-59$ was 2.43 (95\% CI 2.27 to 2.60); the protective effect of marriage appeared stronger for women that is, the respective ORs for women were 3.00 (2.82-3.19) and 3.05 (2.76-3.37). The odds of CF1 by marital status increased and were strongest in younger ages-for example, OR for men aged 30-59 in 2000-04 was $1.54(1.34-1.77)$ and for women was 1.45 (1.26-1.67). The odds of CF2 increased over time (OR in 2000-04 was $1.75(1.61-1.90))$ but did not differ by age, sex or deprivation. Conclusions Marriage is beneficial to survival after a first AMI. This relationship differs by socio-economic and demographic circumstances. The benefits of being married may be due to stronger social support-for example, relationship with short-term case-fatality may be explained by married patients taking less time to seek medical attention and may be explained long-term by higher uptake/commitment to secondary prevention programmes.

\section{CAUSAL EFFECTS OF COX-2 SELECTIVE INHIBITORS RELATIVE TO NON-SELECTIVE NON-STEROIDAL ANTI- INFLAMMATORY DRUGS ON GASTROINTESTINAL BLEEDING AND ACUTE MYOCARDIAL INFARCTION: AN INSTRUMENTAL VARIABLE ANALYSIS}

doi:10.1136/jech.2010.120956.8

N M Davies, G Davey-Smith, F Windmeijer, R M Martin. Department of Social Medicine, University of Bristol, UK

Objective To compare ordinary least squares (OLS) and instrumental variable (IV) estimates of the effects of COX-2 selective inhibitor non-steroidal anti-inflammatory drugs (COX-2s) relative to nonselective NSAIDs (non-selectives) on incidence of gastrointestinal (GI) bleeding, and acute myocardial infarction (MI). We test the validity of the IV estimates, and compare to estimates from randomised controlled trials (RCTs)

Design Cohort study using administration data.

Setting Primary care.

Participants 93562 patients aged over 65 years attending one of 104 general practices (GP) in the UK.

Main outcomes GI bleeding and acute MI

Methods We use data from the UK general practice research database. Our outcomes are incident gastrointestinal bleeding and myocardial infarctions within 60,120,180 and 360 days of the first prescription. We estimate risk differences in outcomes using OLS and IVs, using physician prior prescribing as an IV, and compare them to RCT estimates.

Results The OLS estimates suggested COX-2s were positively associated with GI bleeds, and were negatively associated with MI. Adjusted OLS estimates were attenuated. In contrast our IV estimates imply $-0.43(-1.54,0.68)$ fewer GI bleeds per 100 people treated with COX-2s after 180 days. The IV estimates provide little evidence, $-0.55(-1.2,1.5)$, of a difference in MI between treatments. These are comparable to previously published IV estimates and RCT evidence. 
Conclusions Deriving causal treatment effects using observational health data has proven to be problematic. We demonstrate that in contrast to IV based estimates, conventional OLS methods fail to estimate casual effects as indicated by RCTs.

\section{Childhood and mental health 009 CHRONIC ILLNESS AND EMOTIONAL AND BEHAVIOURAL
STRENGTHS AND DIFFICULTIES IN IRISH CHILDREN}

doi:10.1136/jech.2010.120956.9

${ }^{1,2} \mathrm{U}$ Reulbach, ${ }^{1} \mathrm{~T}$ O'Dowd, ${ }^{3} \mathrm{C}$ McCrory, ${ }^{3} \mathrm{R}$ Layte. ${ }^{1}$ Department of Public Health and Primary Care, Trinity College Centre for Health Sciences, Dublin, Republic of Ireland; ${ }^{2}$ HRB Centre for Primary Care Research, Dublin, Republic of Ireland; ${ }^{3}$ Economic and Social Research Institute (ESRI), Dublin, Republic of Ireland

Objective Childhood chronic illness can have a high impact on the child's quality of life. The foundations of health are established in early life, and are shaped by biological, psychosocial, spiritual and environmental processes and influences. The objectives of this presentation are twofold: firstly to describe the prevalence of chronic illness in 9 -year-olds in Ireland, secondly to illustrate how chronic illness influences the psychological and social development of the children. Methods Analysis was based on data of 8570 9-year old children, and their families who participated in Growing Up in Ireland-the National Longitudinal Study of Children. The sample was generated through the primary school system. A representative sample of 910 schools participated; the sample of children and their families was randomly selected from within the schools. Questionnaires were administered in schools; and after completion of this phase, the project interviewers visited the families of the 9-year olds in their homes and administered core questionnaires to the Study Child and his/her carers who provided either home-based or centre-based care on a regular basis.

Results The overall prevalence of chronic illness reported by mothers among the 9-year old cohort was 11\% (gender-specific prevalence for boys: $13 \%$, and significantly lower for girls: $10 \%)$. Respiratory illnesses accounted for almost half (46\%) of all chronic illnesses, followed by mental and behavioural conditions with 19\% (high gender dysbalance: reported for boys in $24 \%$, for girls in $12 \%$ ). Children with a reported chronic illness had significantly more emotional, conduct, hyperactivity, peer-based and prosocial difficulties when compared with children without a reported chronic illness. Abnormal scores, based on the Strengths and Difficulties Questionnaire were found in $20 \%$ in children with a chronic disease, and in $5.5 \%$ in children without a chronic disease. Socio-economic Status was associated with poorer health outcomes. Furthermore, primary care givers' views and perceptions regarding the chronic illness of the child were found to be a significant factor on child strengths and difficulties in a multivariate model.

Conclusions The majority of 9-year old children can be assessed as healthy. The most common chronic illness in this large cohort was respiratory disease and overall, chronic conditions were found to have a negative impact on the child's emotional and social state.

\section{PARENTAL SEPARATION AND PSYCHOLOGICAL DISTRESS IN EARLY ADULTHOOD: HAS THE EFFECT REDUCED OVER TIME? EVIDENCE FROM TWO BRITISH BIRTH COHORT STUDIES}

doi:10.1136/jech.2010.120956.10

R Lacey, M Bartley, H Pikhart, N Cable, M Stafford. Department of Epidemiology and Public Health, University College London, London, UK

Background An association between parental separation occurring during childhood and psychological distress has been shown many times. UK divorce rates have increased rapidly since the mid-twentieth century. The "reduced effect hypothesis" suggests that the effect of parental separation may have reduced over time as separation has become more common and consequently less stigmatising. Previous studies have looked at this using outcomes of educational attainment, psychological distress in mid-adulthood and receipt of welfare benefits, finding that the effect has not reduced over time; however the effect upon psychological distress in early adulthood has not yet been investigated.

Objective To examine whether the effect of parental separation occurring during childhood on psychological distress in early adulthood has reduced over time, and whether this differs for men and women.

Data, participants and variables This study uses data on 9064 and 6906 participants of the 1958 National Child Development Study (NCDS) and 1970 British Cohort Study (BCS70), respectively. Five sweeps of each study were used - birth, age 5/7, age 10/11, age 16 and age $23 / 26$ years. Parental separation was measured from 0 to 16 years and psychological distress was measured using Rutter's Malaise Inventory at age 23 (NCDS) or age 26 (BCS70), treated as a binary variable $(0-7=$ no distress, $8-24=$ distress $)$. Mental illness in the family was treated as a confounder.

Statistical methods Logistic regression was used to test the association between parental separation and psychological distress in either cohort, both unadjusted and adjusted for confounders, using a pooled data set of both cohorts. Period changes were assessed by testing a cohort-separation interaction. Analyses were conducted separately for men and women.

Results $9.4 \%$ of NCDS participants experienced parental separation in comparison to $21.0 \%$ of BCS70 participants. After adjusting for confounders parental separation increased the odds of reporting psychological distress in NCDS men (OR 2.18, 95\% CI 1.34 to 3.54 ) and women (OR 1.48, 95\% CI 1.05 to 2.08) and this did not differ by gender $(p=0.11)$. Parental separation was not associated with psychological distress in BCS70 adjusted analyses for men or women. However cohort-separation interactions were not statistically significant (men: $p=0.11$, women: $p=0.43$ ).

Conclusions Despite finding an effect of separation in the NCDS and not in the BCS70, the cohort-separation interactions tested were statistically insignificant. The findings of this study therefore imply that the impact of parental separation has not changed over time ("reduced effect hypothesis" is not supported) and that men and women are affected equally.

\section{GENDER DIFFERENCES IN THE EFFECT OF BREAST FEEDING ON ADULT PSYCHOLOGICAL WELL-BEING}

doi:10.1136/jech.2010.120956.11

N Cable, M Bartley, A McMunn, Y Kelly. Department of Epidemiology and Public Health, University College London, UK

Objective To examine the changes in the social distribution of breast feeding and its effect on the psychological well-being of adults via the pathway of childhood psychological health.

Design Prospective cohort study.

Setting We used two British Birth Cohort Studies: National Child Developmental Study (NCDS, born in 1958) and 1970 British Birth Cohort Study (BCS70, born in 1970).

Participants Those who completed information on childhood data (breast feeding, mother's educational level, parenthood at birth, presence of older sibling, and psychosocial adjustment) and midadulthood (psychological ill health and self-efficacy) were included in this study (NCDS: $\mathrm{N}=7750$; BCS70: $\mathrm{N}=6492$ ).

Main outcome measure Childhood psychosocial adjustment was measured by the Bristol Social Adjustment Guides for the NCDS (collected at age 11) and the Rutter scale graded by a teacher for the 